\title{
Towards Simulation-Based Similarity of End User Browsing Processes
}

\author{
Sudhir Agarwal ${ }^{1}$ and Martin Junghans ${ }^{2}$ \\ 1 Stanford Computer Science Department, Stanford University \\ 353 Serra Mall, Stanford, CA-94301, USA \\ sudhir@cs.stanford.edu \\ 2 Institutes AIFB and KSRI, Karlsruhe Institute of Technology \\ Englerstr. 11, 76131 Karlsruhe, Germany \\ junghans@kit.edu
}

\begin{abstract}
For increasingly sophisticated use cases an end user needs to extract, combine, and aggregate information from various (often dynamic) web pages from different websites. Current search engines do not focus on combining information from various web pages in order to answer the overall information need of the user. Semantic Web and Linked Data usually take a static view on the data and rely on providers cooperation. Web automation scripts, initially developed for testing websites, allow end users to capture their browsing activities as executable processes and share them with other end users. A script can contain instructions for accessing, extracting and merging (dynamic) information from various websites for a particular purpose. Techniques for allowing users to search for scripts that satisfy complex constraints restrict to existing scripts in the repository, i.e. they do not deduce scripts that may satisfy the request as well. In this paper, we show how semantic descriptions of web sites can be derived from such scripts, and how such semantic descriptions of web sites along with usage information present in the scripts can be used to obtain new scripts with similar functionality.
\end{abstract}

\section{Introduction}

For many practical purposes end users need information that is scattered across multiple websites. Static websites can be reached and their content can be indexed by the crawlers of state of the art search engines. However, in many cases, end users still require to do a lot of manual work to compile together the required information. Consider for example an end user who is interested in knowing the names of the chairs of a particular track at the previous WWW conferences. As of today Google does not deliver satisfactory results for queries similar to "track chairs of all WWW conferences". Search engines focus on finding individual highly ranked web pages and not on providing the required information directly. Search engines results often contain links to web pages with similar content even though the information need of the user might require pages with complementary information. As a result, an end user needs to pose multiple queries to a search engine, browse through the hits, and aggregate the required information fragments outside of the found web pages. The case of dynamic websites to access the information in the Deep Web [1] is even more complex and still an open challenge

F. Daniel, P. Dolog, and Q. Li (Eds.): ICWE 2013, LNCS 7977, pp. 216-223, 2013.

(C) Springer-Verlag Berlin Heidelberg 2013 
for search engines since it is hard for automatic crawlers to sensibly interact with the dynamic websites. Furthermore, indexing such information is not a suitable technique since the information underlying dynamic websites changes so rapidly that the index becomes quickly outdated.

In contrast to search engine crawlers, end users are able to reach the dynamic web pages. Information retrieval has focused on analyzing such click trails of millions of end users mainly for the purpose of improving web search results. Click trails can be used as endorsements to rank search results more effectively [2], trail destination pages can themselves be used as search results [3], and the concept of teleportation can be used to navigate directly to the desired page [4]. Similarly, large-scale studies of web page revisitation patterns [5] focus on how often users revisit the same page, while ignoring how people get there. The statistics based click analysis methods typically do not consider semantics of user queries and pages. As a result, a frequently used and thus recommended path may not necessarily satisfy the information need, and end users still require to figure out themselves which of the recommended web pages are actually relevant for them and which interactions are required with which web pages.

Semantic Web [6] has proposed the annotation of web pages in order to describe the information content of web pages. However, apart from the fact that still most of the web pages are not annotated, it is hard to build a server-sided semantic information search engines since a crawler will be unable to reach and index the semantic annotations within deep web pages. Linked Data [7] separates the structured data from the traditional web (and as a result also from the end users) completely. The Linked Data approach is primarily useful for application developers since end users cannot be expected to consume RDF directly. Therefore, end users still require human understandable applications to interact with. Semantic Web and Linked Data approaches (i) heavily rely on the availability of structured data, and (ii) providers are expected to provide access to their data through APIs, which is unlikely to happen for valuable data, and (iii) the data made accessible by a provider is often not semantically aligned that of other providers and its usage is restricted, e.g. for advertisement purposes only.

Approaches for searching navigational plans, e.g., [8] cannot compute navigational paths that consist of data flow between web pages, which are not connected in the web graph as well as require a mediated schema. In contrast, web scripts that combine different data sources can introduce and model additional links (like data exchange between different web pages).

Recently, browsing scripts, initially developed for the purpose of testing web sites, have drawn a lot of attention as they can be very useful for the end users as well, especially when end users share their scripts with other end users. In our previous work [9] we have shown how browsing processes satisfying complex user constraints can be efficiently retrieved from a repository of browsing processes. However, the search technique is limited to the directly known scripts as it does not deduce new scripts that may have the required functionality.

Our Approach: We aim at providing end users with a list of browsing processes such that each browsing process in the list will lead an end user to the required information. Our approach build on the idea of end users sharing their browsing processes with other end users so that a large number of browsing process are available for end users to 
choose from. Specifically in this paper our aim is to equip existing browsing process search with the ability to deduce new scripts. We achieve this by providing a technique for computing browsing processes similar to a given browsing process. We first show how user browsing processes (consisting of link selection, form inputs, and information extraction steps) can be formalized. Then we present a method to automatically derive semantic annotations of websites from the browsing processes. Then, we show how functionally similar browsing processes can be generated from the known browsing processes. The computation of the set of all similar browsing processes is done offline, i.e. prior to searching.

\section{Formalization of End User Browsing Processes}

In this section, we present how end user browsing processes can be described formally. The formalization enables generic automatic procedures such as for verification, testing, search and composition. Browsing processes capture users' interactions with websites and local operations. In order to be able to construct browsing processes for a given need we need a formal and semantic model of websites. In contrast to top-down semantics based approaches that require semantically annotated websites, we show how semantic annotations of websites can be derived from browsing processes.

An end user browsing process is a sequential process that coordinates the execution of multiple websites. An end user has a local knowledge base, and the browsing activities that an end user carries out can be categorized into input, output, and local (wrt. the end user knowledge base) actions. An input action causes addition of knowledge from a website into the knowledge base, the output activity emits (without deleting) knowledge from the knowledge base to a website, and a local action causes changes in the knowledge base independent of the websites such as deletion or alignment of knowledge. Such browsing processes can be easily modeled by a process algebra such as $\pi$-calculus [10] with the syntax $\mathbf{0}|c[\mathbf{x}] . P| c\langle\mathbf{y}\rangle . P \mid \tau . P$, where $\mathbf{0}$ denotes the process that does nothing and used as termination symbol, $c[\mathbf{x}] . P$ denotes a process that inputs some values along the channel $c$, binds them to $\mathbf{x}$, and then behaves like the process $P, c\langle\mathbf{y}\rangle . P$ denotes a process that outputs values $\mathbf{y}$ along the channel $c$, and then behaves like the process $P$, and finally $\tau$.P denotes a process that performs a local action, and then behaves like the process $P$. A local action is an action performed by the end user in his/her local knowledge base in order to structure the knowledge as per user's needs. The set of local actions available to an end user depends on the data model of the knowledge base, e.g. a relational model will allow different operations than a graph based model.

In a pure process algebra such as the $\pi$-calculus the process resources and variable are seen as strings without any structure. As a result, it is hard for an end user to understand which values he or she should provide for the variables in order to get the desired result. We fill this gap by allowing process variables to have semantics with the help of a domain ontology $O_{D}$ expressed in $\mathcal{A L C}$ (attributive concept language with complements) [11]. E.g., input parameters $\mathrm{x}$ and the communication channel $\mathrm{c}$ of an input activity are process resources and further described in $O_{D}$. With $\mathcal{A L C}$ we can describe not only the types of process variables but also their relationships with other 
process variables. For example, if an input activity has two parameters of type 'Person', we can also describe that the first person should be father of the second person. Precisely, the local knowledge modeled as $\mathrm{ABox}$ of $\mathcal{A L C}$ can be modified by adding or removing following types of axioms: (i) add sameAs relation between two individuals, (ii) add typeOf relation between an individual and a concept, (iii) domain specific relationships between two individuals (object properties), and (iv) relationships between an individual and a literal (data properties) .

Example 1. The WWW 2013 conference website at URL Www2 013 contains a link to the call for research papers web page www2013/cfp that provides links of the form www2013/cfp/trackname for all the research tracks of the conference. Selecting one of the provided links returns the page about the track that lists topics, chairs, and PC members of the track. The second website is the DBLP at $\mathrm{dblp}$ that among other information on the entry page contains the web form $\mathrm{dblp} / \mathrm{search}$ for searching for publications of an author. The form $\mathrm{dbl} p /$ search takes the author name as single input value and returns a page with the list of publication of the author.

A researcher who is interested in submitting a research paper to the "Bridging Structured and Unstructured Data" track wishes to know more about the research background of the track chairs. For this purpose he wants to have a list of most recent 3 journal articles of the track chairs. For this purpose, the researcher performs the following navigation actions (i) visit www2013/cfp, (ii) click on www2013/cfp/bridging, (iii) extract the names $t c_{1}, \ldots, t c_{k}$ of the track chairs, (iv) visit $\mathrm{dblp}$, (v) for each track chair $t c_{i}$ enter $t c_{i}$ in the form $\mathrm{dblp} / \mathrm{search}$ and extract the publication of $t c_{i}$. In our formalism this browsing steps can be formalized as a process as:

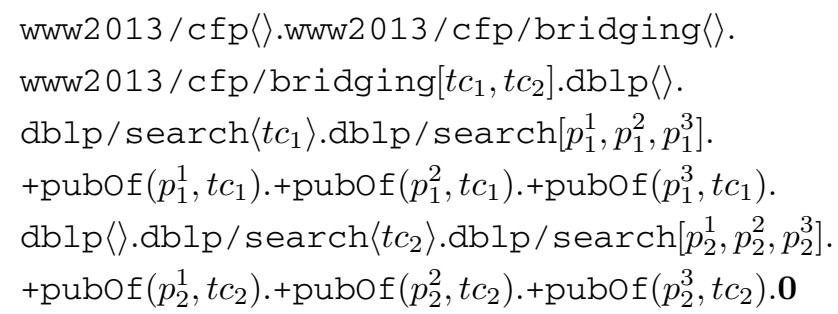

As shown in the above example end users' actions for integrating the extracted knowledge with his/her local knowledge base are part of the browsing process. Note that an end user has direct incentives for keeping his/her knowledge base consistent since it makes it easier to query already visited information much faster and in structured way, e.g. with SPARQL. If the domain ontology used for structuring his/her knowledge base is a shared vocabulary among a group of end users, the web browsing processes become easily reusable within the group.

Definition 1. In an expression of form $x[y]$.P the occurrence of $y$ is a binding occurrence and in each case the scope of the occurrence is $P$. An occurrence of $y$ in a browsing process is said to be free if it does not lie within the scope of a binding occurrence of $y$. The set of names occurring free in $P$ is denoted by $f n(P)$.

Definition 2. For a set of atomic propositions $P$ and a set of actions $A$, a labeled transition system (LTS) is a tuple $(S, T, A, \lambda)$, where $S$ is a finite set of states, $T \subseteq S \times A \times S$ 
a set of labeled transitions between the states, and $\lambda: S \rightarrow 2^{A P}$ a labeling function that maps each set $s \in S$ to the set of atomic propositions that are true in $s$.

Formal semantics of a browsing process is defined by a mapping to an LTS that is a finite linear sequence of states connected by transitions. A state represents the content of the knowledge base at a point of time, and a transition an input, output, or local action. The mapping is done by applying the following three rules:

$$
\frac{-}{\tau . P \stackrel{\tau}{\rightarrow} P}, \frac{-}{x\langle y\rangle . P \stackrel{x\langle y\rangle}{\rightarrow} P} \text {, and } \frac{-}{x[z] . P \stackrel{x[w]}{\rightarrow} P\{w / z\}} \text { with } w \notin f n(P) \text {. }
$$

The main idea for obtaining the LTS for a given process expression lies in viewing the process expressions as states and applying the rules on the states to obtain next states. E.g., the first rule states with the nominator - that a silent action does nor require any preconditions to fire, and with the denominator $\tau . P \stackrel{\tau}{\rightarrow} P$ that the process evolves from state $\tau$.P to state $P$ by performing the silent action. Analogous for the other two rules.

\section{Computation of Similarity between Browsing Processes}

Our overall aim is to find known and similar processes efficiently as well as rank them and to provide the ranked list of appropriate browsing processes to an end user in a same way search engines presents their results. For this purpose, we first compute semantic similar websites. A website usage in a browsing process can be replaced by the usage of a semantically similar website in order to obtain a new browsing process that provides the similar functionality. In this paper, we only deal with the problem of similarity computation.

\subsection{Derivation of Semantic Description of Websites}

A website can be seen as a sequential process. It outputs pages to and receives inputs from a user through her web browser. Similar to an end user performing local operations in her knowledge base, a web server can perform local operations, e.g., by invoking CGI scripts or servlets.

A web page is a message sent by a server. In addition to the information content, a web page offers a choice of links and forms. The links and the action URLs of the forms refer to external or internal locations wrt. the web server. Sometimes, a web page may contains an HTTP redirect instruction to redirect the browser to another URL. The output action of the server that produces a web page with URL $u$ with $l$ values $v_{1}, \ldots, v_{l}, m$, links $l_{1}, \ldots, l_{m}$ and $n$ forms $f_{1}, \ldots, f_{n}$ is described as $u\left\langle v_{1}, \ldots, v_{l}, l_{1}, \ldots, l_{m}, f_{1}, \ldots, f_{n}\right\rangle . P$, where $P$ denotes the web server process (may be simply 0 ) after outputting the web page.

Form submission and link selection are similarly modeled except that a user has the option of filling in values in the former case. We model the arguments of a link as classes and the values of the arguments as instances of the corresponding classes of the ontology associated with the website. The semantic annotation of the link arguments also allow specification of relationships of arguments with other arguments or other 
ontology elements. The input parameters of a form are described in a similar fashion. The name of the ontology class corresponding to the range of a value can be often derived from the id or label of the input field (see e.g. [12]). Note that we require only the existence of an id or a label. They ids and labels may or may not be semantically meaningful. Some form input types provide a set of predefined values from which one or more values may be selected. In these cases, the provided values are modeled as ontology instances, while the class representing the range of an input field as an enumeration class instead of a normal class. Execution semantics of the formalism for describing websites is defined by mapping its syntax to an LTS that is constructed by applying the rules of execution semantics [13]. The states of the LTS correspond to the knowledge of the process in that stage of the execution, and the transitions correspond to the atomic (input, output, or local) activities. There exists decidable reasoning procedures for LTSs in which the ABox of the ontology may change but the TBox of the ontology may not change during the execution [14].

Having the formal models of browsing processes and websites, we now present the derivation of semantic descriptions of websites from a given set of browsing processes. The main insight underlying such a derivation is that an output of a browsing process corresponds to an input of a web form, and an input of a browsing process corresponds to an output of a web form.

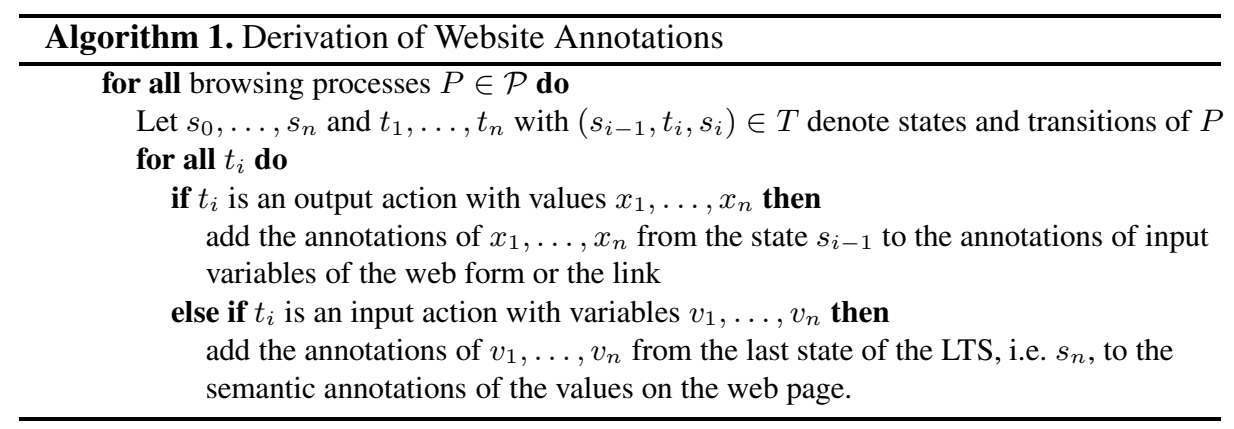

Algorithm 1 describes the derivation of semantic annotations of websites from the information appearing in the browsing processes. Note that due to the chosen level of abstraction, link selections and form submissions are treated similarly and it is not possible to detect whether an output activity of a browsing process corresponds to a link selection or a form submission. However, this does not create any obstacles in our approach. If a distinction of links and forms is desired the browsing process language can be enhanced to add these information. A good heuristic could be to consider the output activities without arguments as as link selections.

\subsection{Computing Similar Websites}

From the generic description of websites, we compute the semantic similarity of websites as follows: A website $w_{1}$ simulates another website $w_{2}$, in short $w_{1} \sim w_{2}$, if for every possible input $i$ for $w_{2}$ if $w_{2}$ outputs $o$ then $w_{1}$ also outputs $o$. In order to compute $w_{1} \sim w_{2}$ we need to check (i) that $w_{1}$ accepts every input that $w_{2}$ accepts, and 
(ii) that the output of $w_{1}$ has all the semantic annotations that the output of $w_{2}$ has. This means, the state of $w_{2}$ after input must be a model of the semantic annotations of input parameters of $w_{1}$, and the state of $w_{1}$ at the time of output must be a model of semantic annotations of output parameters of $w_{2}$. Both conditions can be checked by a DL reasoner that supports rules, e.g. HermiT [15] by modeling the states as ABoxes, the semantic annotations as queries, and checking if an ABox is an answer of the query.

\subsection{Computing Similar Browsing Processes}

We compute similar browsing processes of a given browsing process by replacing the usage of websites in the browsing process by the usage of websites that simulate the websites as shown in the Algorithm 2. The algorithm computes in each iteration of the while loop the set $\operatorname{sim}(P)$ of browsing processes that are similar to a browsing process $P$. The termination of the algorithms is guaranteed since the number of browsing processes, the number of websites within a browsing process, and the number of websites to similar to a website are all finite, and the algorithm continues with the next iteration only when it can construct at least one new browsing process.

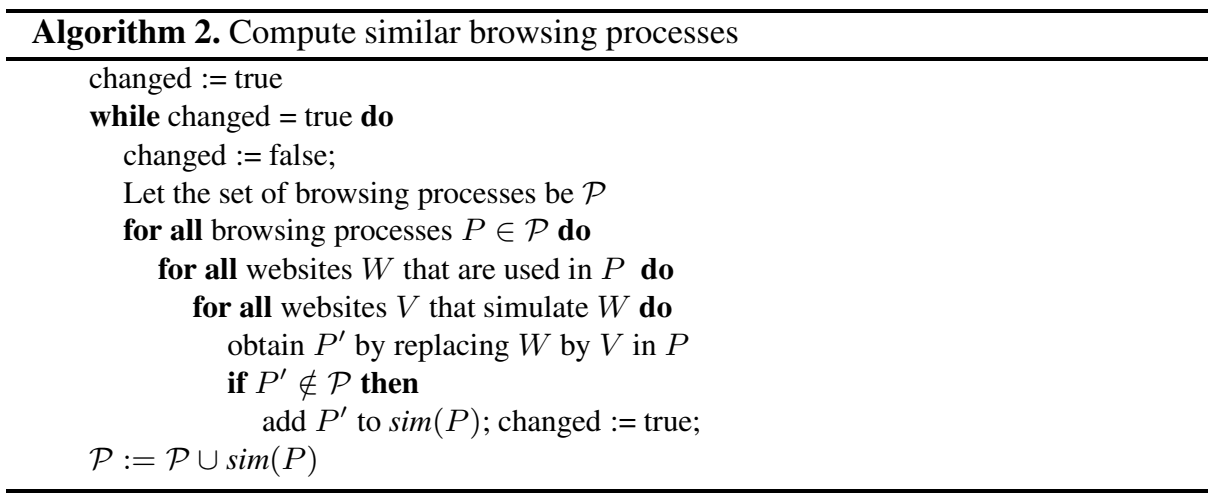

\section{Conclusion and Outlook}

In this paper, we targeted the problem of tediousness of information gathering from various websites from a bottom-up perspective that proposes capturing and sharing of end user browsing processes as opposed to the top-down approach that requires annotated websites in the first place. We have shown how an end user browsing process can be formalized without requiring extra manual effort for declarative semantic annotations, and how processes can be mapped to a labeled transition system. We have further shown how semantic annotations of the web browsing processes can be derived from end user actions. Existing techniques for searching and composing browsing processes could exploit the similarity of browsing processes to deduce new browsing processes and to gain performance respectively. Such an extension of the search technique presented in one our previous work [9] is planned as future work. 
Acknowledgments. The authors acknowledge the support of the European Community's Seventh Framework Programme FP7-ICT-2011-7 (XLike, Grant 288342).

\section{References}

1. Bergman, M.K.: The deep web: Surfacing hidden value. The Journal of Electronic Publishing 7 (2001)

2. Bilenko, M., White, R.W.: Mining the search trails of surfing crowds: identifying relevant websites from user activity. In: Proceedings of the 17th International Conference on World Wide Web, WWW 2008, pp. 51-60. ACM (2008)

3. White, R.W., Huang, J.: Assessing the scenic route: measuring the value of search trails in web logs. In: Proceeding of the 33rd International ACM SIGIR Conference on Research and Development in Information Retrieval (SIGIR), pp. 587-594. ACM (2010)

4. Teevan, J., Alvarado, C., Ackerman, M.S., Karger, D.R.: The perfect search engine is not enough: a study of orienteering behavior in directed search. In: Dykstra-Erickson, E., Tscheligi, M. (eds.) CHI, pp. 415-422. ACM (2004)

5. Adar, E., Teevan, J., Dumais, S.T.: Large scale analysis of web revisitation patterns. In: Proceedings of the SIGCHI Conference on Human Factors in Computing Systems, CHI 2008, pp. 1197-1206. ACM (2008)

6. Berners-Lee, T., Hendler, J., Lassila, O.: The Semantic Web: a new form of Web content that is meaningful to computers will unleash a revolution of new possibilities. Scientific American 5, 34-43 (2001)

7. Bizer, C., Heath, T., Berners-Lee, T.: Linked data - the story so far. International Journal on Semantic Web and Information Systems 5, 1-22 (2009)

8. Friedman, M., Levy, A.Y., Millstein, T.D.: Navigational plans for data integration. In: Hendler, J., Subramanian, D. (eds.) AAAI/IAAI, pp. 67-73. AAAI Press / The MIT Press (1999)

9. Junghans, M., Agarwal, S.: Efficient search for web browsing recipes. In: Proceedings of the 20th International Conference on Web Service (ICWS 2013). IEEE (June 2013)

10. Milner, R., Parrow, J., Walker, D.: A Calculus of Mobile Processes, Parts I and II. Journal of Information and Computation 100, 1-77 (1992)

11. Schmidt-Schauß, M., Smolka, G.: Attributive concept descriptions with complements. Artif. Intell. 48, 1-26 (1991)

12. Madhavan, J., Ko, D., Kot, L., Ganapathy, V., Rasmussen, A., Halevy, A.: Google's deep web crawl. Proceedings of the VLDB Endowment Archive 1, 1241-1252 (2008)

13. Agarwal, S., Lamparter, S., Studer, R.: Making Web services tradable - A policy-based approach for specifying preferences on Web service properties. Web Semantics: Science, Services and Agents on the World Wide Web, Special Issue on Policies 7(1), 11-20 (2009)

14. Gabbay, D., Kurucz, A., Wolter, F., Zakharyaschev, M.: Many-dimensional modal logics: theory and applications. Studies in Logic, vol. 148. Elsevier Science (2003)

15. Motik, B., Shearer, R., Horrocks, I.: Hypertableau Reasoning for Description Logics. Journal of Artificial Intelligence Research 36, 165-228 (2009) 